\title{
Two Loop Partially Quenched and Finite Volume Chiral Perturbation Theory Results
}

\author{
Johan Bijnens* \\ Department of Theoretical Physics, Lund University, Sölvegatan 14A, SE 22362 Lund, Sweden \\ E-mail: bijnensethep.lu.se
}

\section{Niclas Danielsson}

Department of Theoretical Physics, Lund University, Sölvegatan 14A, SE 22362 Lund, Sweden and

Division of Mathematical Physics, LTH, Lund University, Box 118, S 22100 Lund, Sweden E-mail: niclas.danielssonematfys.lth.se

\section{Karim Ghorbani}

Department of Theoretical Physics, Lund University, Sölvegatan 14A, SE 22362 Lund, Sweden E-mail: karimethep.lu.se

\section{Timo Lähde}

Department of Theoretical Physics, Lund University, Sölvegatan 14A, SE 22362 Lund, Sweden E-mail: talahdedthep.lu.se

\begin{abstract}
This talk presents some results relevant for lattice QCD at higher order in ChPT. First we discuss the finite volume corrections at two loops for the quark condensate as well as a Lüscherlike finite volume formula for it. The latter allows for an alternative determination of meson sigma terms. The second set of results presented here are the calculations at two loops in partially quenched chiral perturbation theory of masses and decay constants of the charged mesons. We present results for all relevant quark mass combinations for the cases with two and three sea quarks.
\end{abstract}

XXIIIrd International Symposium on Lattice Field Theory

25-30 July 2005

Trinity College, Dublin, Ireland

\footnotetext{
* Speaker.
} 


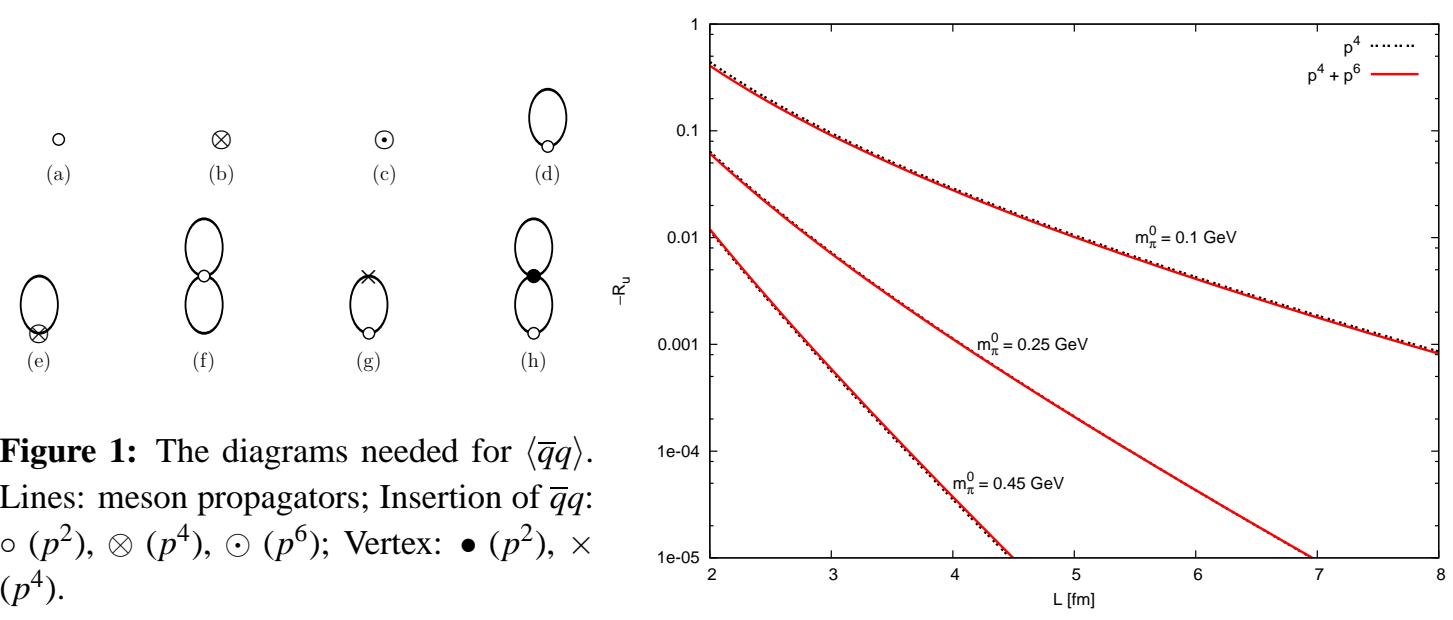

Figure 2: The finite volume corrections $R_{u}$ to $\langle\bar{u} u\rangle$.

\section{Introduction}

In this talk we present some work useful for the extrapolation of lattice QCD numerical results to the physical volume and quark masses. At low energies, there exists an effective fi eld theory approximation to QCD known as Chiral Perturbation Theory (ChPT) [1]. We can thus use this theory to fi t to lattice QCD numerical results and then extrapolate to infi nite volume and the physical quark masses. ChPT is based on the correct inclusion of the nonanalytic structure in all amplitudes due to the Goldstone bosons of spontaneous chiral symmetry breaking and all relations that follow from the chiral Ward identities. This is done in a systematic expansion in momenta, energies and quark masses. There exists an enormous body of work in ChPT. Meson masses, decay constants and vacuum expectation values at infi nite volume are known to two-loop order, see [3, 3, 4] and references therein. In this talk we present the extension of these results in two directions, fi nite volume for vacuum expectation values and partial quenching for the masses and decay constants.

\section{2. $\langle\bar{q} q\rangle$ at Finite Volume}

The work presented in this section will be published in [5]. At fi nite volume, particles can propagate around the world. Taking this into account in ChPT was first done in [6]. Many more quantities have since been calculated at the one-loop level. Here we would like to address this problem at the two-loop level. A major problem is to evaluate the necessary loop integrals at twoloop order which has not been done so far $^{1}$. An alternative approach to fi nite volume was pioneered by Lüscher [7]. Here the leading effect of a fi nite volume is taken into account by an integral over another amplitude. An overview of earlier fi nite volume work can be found in [8].

We would like to check how well Lüscher's method works when the fi nite volume quantities are fully calculated. At one loop in ChPT the full results for many quantities can be derived using Lüscher's method. We therefore want to study a quantity at two-loop level.

There are a few quantities where the two-loop calculation does not involve irreducible twoloop integrals. One such example is precisely the value of $\langle\bar{q} q\rangle$ as can be seen from the diagrams

\footnotetext{
${ }^{1}$ Some partial results were presented by C. Haefeli at this conference.
} 


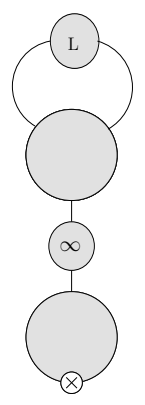

(a)

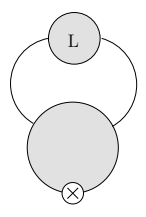

(b)

Figure 3: The two classes of contributions to a Lüscher formula for a vacuum expectation value. The label $L$ or $\infty$ means a finite volume or infinite volume propagator.

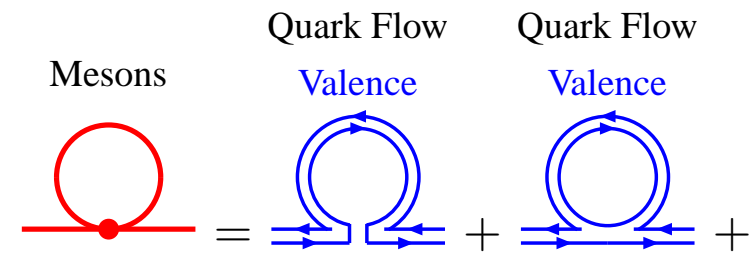

Quark Flow Quark Flow

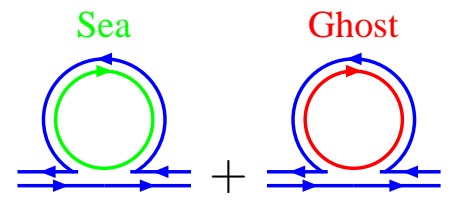

Figure 4: A schematic representation of how a meson loop contribution has quark flows with valence, sea and ghost quark closed loops present.

shown in Fig. 1. $\langle\bar{q} q\rangle$ at two-loops at infi nite volume is known [3]. We have redone this calculation checking explicitly that the $\mathscr{O}(d-4)$ parts of the integrals do not contribute to the fi nal results. The known one-loop integrals at fi nite volume are therefore suffi cient. Numerical results for $R_{q}=$ $\left(\langle\bar{q} q\rangle_{V}-\langle\bar{q} q\rangle_{\infty}\right) /\langle\bar{q} q\rangle_{\infty}$ are shown in Fig. 国 for the case of the up quark condensate. We have here expressed the masses appearing in the integrals as the lowest order masses, leading to very small $\mathscr{O}\left(p^{6}\right)$ corrections.

The analogue of Lüscher's mass formula is derived in [5]. The two types of contributions are shown in Fig. 3. Those of Fig. Ba do not contribute in ChPT to parity-even operators like $O=\bar{q} q$. Keeping also the non-leading contributions of the same propagator, see [B], we obtain

$$
\begin{aligned}
\langle O\rangle_{V}-\langle O\rangle_{\infty} & =-\sum_{\vec{n} \neq \vec{o}} \frac{1}{16 \pi^{2}} \int_{0}^{\infty} \frac{d q^{2} q^{2}}{\sqrt{m_{0}^{2}+q^{2}}} e^{-\sqrt{\vec{n}^{2}\left(m_{0}^{2}+q^{2}\right) L^{2}}}\langle\phi|O| \phi\rangle \\
& =-\langle\phi|O| \phi\rangle\left(\sum_{k=1, \infty} \frac{m(k)}{16 \pi^{2}} \frac{m_{0}^{2}}{\sqrt{\zeta(k)}} K_{1}(\zeta(k))\right) .
\end{aligned}
$$

with $\zeta(k)=\sqrt{k} m_{0} L$ for the contribution of a neutral scalar $\phi$ to the fi nite volume dependence of $\langle O\rangle . m(k)$ is the number of times $k=\sum_{i=1,3} n_{i}^{2}$ appears in the sum over $\vec{n}$.

Note that in this case there is a universal factor which contains the matrix element $\langle\phi|O| \phi\rangle$ rather than an integral as in the fi nite volume mass formula. This also means that the fi nite volume dependence can be used to obtain this matrix element. Unfortunately, the extremely small $\mathscr{O}\left(p^{6}\right)$ corrections allow no meaningful test of the Lüscher formula versus the full $\mathscr{O}\left(p^{6}\right)$ calculation.

\section{Partially Quenched ChPT at Two Loops}

The extension of ChPT to the quenched and partially quenched case has been done at one loop by Morel, Bernard, Golterman and Sharpe [9, 10] and discussed in detail in [11]. We use here the version without the super-singlet $\Phi_{0}$. Basically one adds bosonic ghost quarks to cancel the effect 
of valence loops and a separate set of sea quarks to the Lagrangian of QCD, shown schematically in Fig. 6 . As a consequence the Lagrangian gets (approximately, see [11]) a graded symmetry $S U\left(n_{v}+n_{s} \mid n_{v}\right) \times S U\left(n_{v}+n_{s} \mid n_{v}\right)$, with $n_{v}$ the number of valence and $n_{s}$ the number of sea quarks, rather than the usual chiral symmetry. We thus expect the low-energy effective theory to be Chiral Perturbation Theory with this symmetry group instead. Most of the classifi cation work and the infi nity structure at two loops [12] can be carried over to this sector by simply replacing traces by super-traces. Exceptions to this are baryons and the Cayley-Hamilton relations.

One important note is that since QCD is a continuous limit of partially quenched QCD (PQQCD) the low-energy constants (LECs) from ChPT can be derived directly from those of PQChPT, e.g.

$$
L_{1}^{r}=L_{0}^{r(3 p q)} / 2+L_{1}^{r(3 p q)}
$$

The superscript $(3 p q)$ means the constants relevant for 3 sea quark flavour PQChPT.

The calculations published so far are: The mass at two loops in PQChPT for the case of equal valence and equal sea-quark masses for three sea flavours [13], the decay constants for three sea flavours [14] and the masses and decay constants for two sea flavours [15]. The remaining mass combinations for the masses are in the process of being written up [16. That article will also contain a longer introduction to PQChPT at two-loop order.

The expressions are extremely long compared to those of the normal case [2]. The main reasons are: First, there are many more masses even compared to the isospin breaking case [4]. This allows for many more mass combinations to show up. Second and most important, the neutral or diagonal sector propagators contain both single and double poles with complicated coeffi cients. These coeffi cients are ratios of differences of lowest order meson masses to various powers. These rational functions of quark masses satisfy many nontrivial relations which allowed to compress the formulas by signifi cantly more than an order of magnitude, and bring them to a publishable size, but they still leave an added layer of complexity.

The double poles in the propagators appear because PQQCD is not a well defi ned fi eld theory but only exists as a thermodynamical model. This leads to the well-known quenched logarithms [ 9 ].

In the remainder we present some numerical results at two-loop order for masses and decay constants. We present them as a function of quark masses, $m_{i}$, via the lowest order meson masses

$$
\chi_{i}=m_{M}^{2(0)} \equiv 2 B_{0} m_{i} .
$$

The border of validity for ChPT is for the $\chi_{i}$ of order $0.25 \sim 0.30 \mathrm{GeV}^{2}$.

One can of course show any amount of fi gures for the various mass cases for decays and masses. We restrict to a few simple cases. First the $1+1$ case, i.e. valence masses equal: $\chi_{1}=\chi_{2}=$ $\chi_{3}$ and sea masses equal: $\chi_{4}=\chi_{5}=\chi_{6}$. We show results along the lines $\chi_{4}=\tan \theta \chi_{1}$ and $\chi_{4}$ constant, shown in Fig. 5 in the valence/sea quark plane. The result for the mass at one-loop order is shown in Fig. 6. Notice the presence of the quenched chiral logarithm along the line labeled A. The same result at two-loop order is shown in Fig. 7. The corrections are much larger than at one-loop order. This is also the case for normal ChPT [4]. There are choices of the LECs possible where this is not so [3, 17]. We also show results for the $1+1$ case of the decay constant [14].

Plots for the other mass cases can be found in the published papers. The analytical form of the expressions can be downloaded from [18] and numerical programs are available from the authors. 


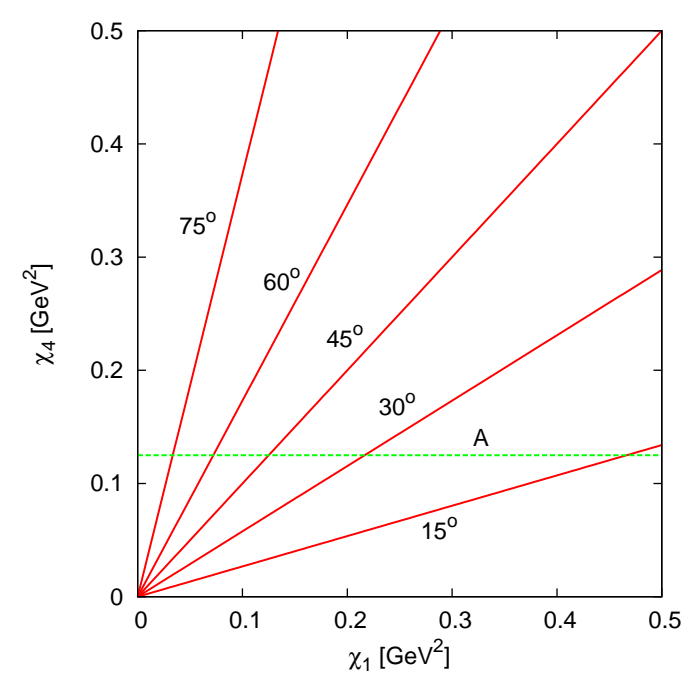

Figure 5: The lines in the sea/valence quark mass along which results are shown in the next figures.

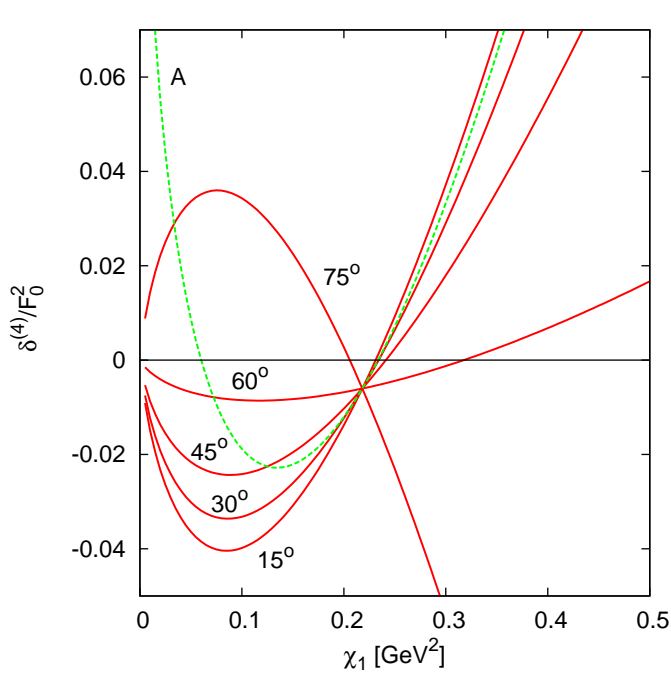

Figure 6: The relative correction to the masses at order $p^{4}$ for the $1+1$ case [13].

In conclusion for this part, we have obtained the two-loop formulae for charged meson masses and decay constants in PQChPT and shown that the corrections can be sizable. We hope that this work will be useful for the numerical lattice QCD community.

\section{Acknowledgments}

This work is supported by the European Union TMR network, Contract No. HPRN-CT-200200311 (EURIDICE) and by the European Community-Research Infrastructure Activity Contract No. RII3-CT-2004-506078 (HadronPhysics). TL acknowledges a Mikael Björnberg memorial

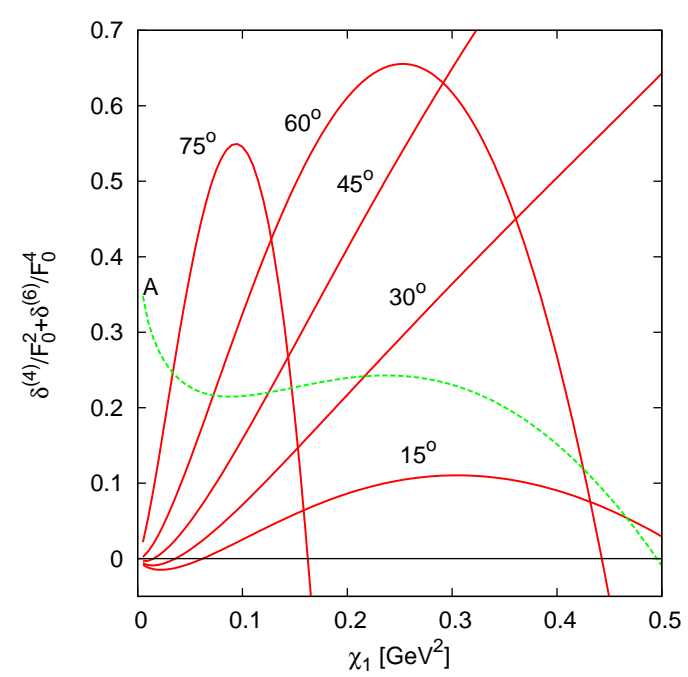

Figure 7: The relative correction to the masses at order $p^{6}$ for the $1+1$ case [13].

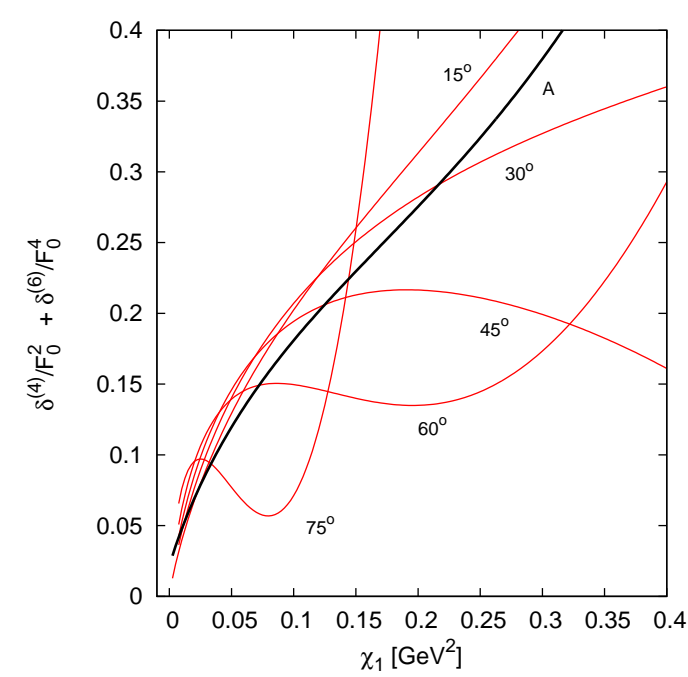

Figure 8: The relative correction to the decay constants at order $p^{6}$ for the $1+1$ case [14]. 
foundation travel grant and $\mathrm{KG}$ an Iranian government fellowship.

\section{References}

[1] S. Weinberg, Phenomenological Lagrangians, Physica A 96, 327 (1979); J. Gasser and H. Leutwyler, Chiral Perturbation Theory To One Loop, Ann. Phys. 158, 142 (1984); Chiral Perturbation Theory: Expansions In The Mass Of The Strange Quark, Nucl. Phys. B 250, 465 (1985).

[2] G. Amorós, J. Bijnens and P. Talavera, Two-point functions at two loops in three flavour chiral perturbation theory, Nucl. Phys. B 568, 319 (2000) [hep-ph/9907264].

[3] G. Amorós, J. Bijnens and P. Talavera, $K_{l 4}$ form-factors and $\pi \pi$ scattering, Nucl. Phys. B 585 (2000) 293 [Erratum-ibid. B 598 (2001) 665] [hep-ph / 0003258 ].

[4] G. Amorós, J. Bijnens and P. Talavera, QCD isospin breaking in meson masses, decay constants and quark mass ratios, Nucl. Phys. B 602 (2001) 87 [hep-ph / 0101127 ].

[5] J. Bijnens and K. Ghorbani, to be published.

[6] J. Gasser and H. Leutwyler, Spontaneously Broken Symmetries: Effective Lagrangians At Finite Volume, Nucl. Phys. B 307, 763 (1988).

[7] M. Lüscher, Volume Dependence Of The Energy Spectrum In Massive Quantum Field Theories. 1. Stable Particle States, Commun. Math. Phys. 104 (1986) 177.

[8] G. Colangelo, Finite volume effects in chiral perturbation theory, Nucl. Phys. Proc. Suppl. 140, 120 (2005) [hep-lat/0409111].

[9] A. Morel, Chiral Logarithms In Quenched QCD, J. Phys. (France) 48, 1111 (1987); C. W. Bernard and M. F. L. Golterman, Chiral perturbation theory for the quenched approximation of QCD, Phys. Rev. D 46, 853 (1992) [hep-lat/9204007]; S. R. Sharpe, Quenched chiral logarithms, Phys. Rev. D 46, 3146 (1992) [hep-lat/9205020].

[10] C. W. Bernard and M. F. L. Golterman, Partially quenched gauge theories and an application to staggered fermions, Phys. Rev. D 49, 486 (1994) [hep-lat/9306005].

[11] S. R. Sharpe and N. Shoresh, Physical results from unphysical simulations, Phys. Rev. D 62, 094503 (2000) [hep-lat/ 0006017$]$, Partially quenched chiral perturbation theory without $\Phi_{0}$, Phys. Rev. D 64, 114510 (2001) [hep-lat/ 0108003 ].

[12] J. Bijnens, G. Colangelo and G. Ecker, The mesonic chiral Lagrangian of order $p^{6}$, JHEP 9902, 020 (1999) [hep-ph/9902437], Renormalization of chiral perturbation theory to order $p^{6}$, Ann. Phys. 280, 100 (2000) [hep-ph/9907333].

[13] J. Bijnens, N. Danielsson and T. A. Lähde, The pseudoscalar meson mass to two loops in three-flavor partially quenched $\chi P T$, Phys. Rev. D 70 (2004) 111503 [hep-lat/ 0406017 ].

[14] J. Bijnens and T. A. Lähde, Decay constants of pseudoscalar mesons to two loops in three-flavor partially quenched $\chi P T$, Phys. Rev. D 71 (2005) 094502 [hep-lat/ 0501014 ].

[15] J. Bijnens and T. A. Lähde, Masses and decay constants of pseudoscalar mesons to two loops in two-flavor partially quenched chiral perturbation theory, to be published in Phys. Rev. D, hep-lat/0506004.

[16] J. Bijnens, N. Danielsson and T. A. Lähde, to be published.

[17] J. Bijnens and P. Dhonte, Scalar form factors in SU(3) chiral perturbation theory, JHEP 0310 (2003) 061 [hep-ph/0307044].

[18] See http://www.thep.lu.se/ bijnens/chpt.html. 